Issued by Sandia National Laboratories, operated for the United States Department of Energy by Sandia Corporation.

NOTICE: This report was prepared as an account of work sponsored by an agency of the United States Government. Neither the United States Government, nor any agency thereof, nor any of their employees, nor any of their contractors, subcontractors, or their employees, make any warranty, express or implied, or assume any legal liability or responsibility for the accuracy, completeness, or usefulness of any information, apparatus, product, or process disclosed, or represent that its use would not infringe privately owned rights. Reference herein to any specific commercial product, process, or service by trade name, trademark, manufacturer, or otherwise, does not necessarily constitute or imply its endorsement, recommendation, or favoring by the United States Government, any agency thereof, or any of their contractors or subcontractors. The views and opinions expressed herein do not necessarily state or reflect those of the United States Government, any agency thereof, or any of their contractors.

Printed in the United States of America. This report has been reproduced directly from the best available copy.

Available to DOE and DOE contractors from

Office of Scientific and Technical Information

P.O. Box 62

Oak Ridge, TN 37831

Prices available from (703) 605-6000

Web site: http://www.ntis.gov/ordering.htm

Available to the public from

National Technical Information Service

U.S. Department of Commerce

5285 Port Royal Rd

Springfield, VA 22161

NTIS price codes

Printed copy: $\mathrm{A03}$

Microfiche copy: A01

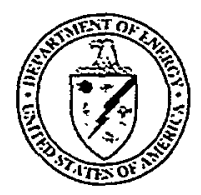




\section{DISCLAIMER}

Portions of this document may be illegible in electronic image products. Images are produced from the best available original document. 
SAND99-1480

Unlimited Release

Printed June 1999

\title{
The ASCI Network for SC '98: Dense Wave Division Multiplexing for Distributed and Distance Computing
}

\author{
Thomas J. Pratt, Luis G. Martinez, and Michael O. Vahle \\ Advanced Network Integration \\ Roger L. Adams \\ Telecommunications Operations Department II \\ Sandia National Laboratories \\ P. O. Box 5800 \\ Albuquerque, NM 87185-0806 \\ Wayne Butman \\ Lawrence Livermore National Laboratory \\ University of California \\ P. O. Box 808 \\ Livermore, California 94550
}

\begin{abstract}
This document highlights the DISCOM ${ }^{2}$ 's Distance computing and communication team activities at the 1998 Supercomputing conference in Orlando, Florida. This conference is sponsored by the IEEE and ACM. Sandia National Laboratories, Lawrence Livermore National Laboratory, and Los Alamos National Laboratory have participated in this conference for ten years. For the last three years, the three laboratories have a joint booth at the conference under the DOE's ASCI, Accelerated Strategic Computing Initiatives. The DISCOM communication team uses the forum to demonstrate and focus communications and networking developments. At SC '98, DISCOM demonstrated the capabilities of Dense Wave Division Multiplexing. We exhibited an OC48 ATM encryptor. We also coordinated the other networking activities within the booth. This paper documents those accomplishments, discusses the details of their implementation, and describes how these demonstrations support overall strategies in ATM networking.
\end{abstract}




\section{CONTENTS}

LIST OF FIGURES

1 INTRODUCTION 4

2 SC '98 NETWORKS

3 NETWORK DESIGN $\quad 7$

4 DENSE WAVE DIVISION MULTIPLEXING DEMONSTRATION $\quad 8$

5 EXTENDING THE SNL EON WITH ATM 11

6 REMOTE VISUALIZATION USING COMPRESSED ATM VIDEO 11

7 ACCELERATED DATA TRANSFER WITH TCP/IP PARALLELIZED FTP13

8 LESSONS LEARNED 16

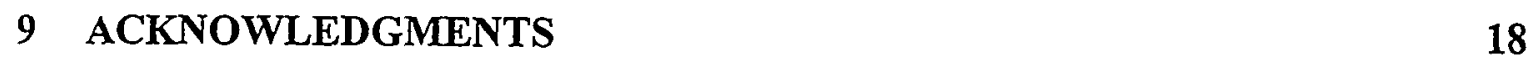

$\begin{array}{llr}10 & \text { REFERENCES } & 19\end{array}$ 


\section{List of Figures}

FIGURE 1: ASCI BOOTH LAYOUT

FIGURE 2: THE CABLING LAYOUT

FIGURE 3: CONFIGURATION OF ROUTER AND SWITCH IN THE PRODUCTION ASCI NETWORK

FIGURE 4: DWDM DEMONSTRATION PHYSICAL SETUP

FIGURE 5: SC '98 DEMONSTRATION SETUP

FIGURE 6: HPSS TRANSFER RATE GRAPH

FIGURE 7: A PARALLEL NETWORKING INFRASTRUCTURE 


\section{Introduction}

SC '98 marked the tenth year for IEEE's high performance computing and communication conference. The advanced networking department at Sandia National Laboratories has used the annual supercomputing conference sponsored by the IEEE and $\mathrm{ACM}$ for the past several years as a forum to demonstrate and focus communication and networking developments. The string of participation began in Minneapolis at the 1992 conference with a demonstration of the prototype Switched Multi-megabit Data and Synchronous Optical Network (SONET) technology that Sandia eventually used in its consolidation of supercomputers [1]. Starting at SC '96 in Pittsburgh, Pennsylvania, Sandia National Laboratories, Lawrence Livermore National Laboratory and Los Alamos National Laboratory have jointly participated in the conference under the ASCI banner. Working together at the conference has furthered the ASCI vision of tightly coupling the HPC activities within the national laboratories. This year continued with the cooperative effort with the joint participation from Sandia National Laboratories and Lawrence Livermore National Laboratory in the planning and support of the booth's production network.

The networking activities this year were extensive. The DISCOM team demonstrated DWDM, Dense Wave Division Multiplexing, as a way to provide parallel gigabit per second communication for distance high performance computing. Also demonstrated were a Sandia developed gigabit per second encryption hardware, Parallel FTP, Scientific Visualization using gigabit Ethernet and visualization using MJPEG ATM. The ASCI booth's SCINET connection for the first time exceeded a gigabit/sec data rate. The connection to SCINET this year was an OC48 ATM circuit. Also present, within the ASCI booth, was gigabit Ethernet. Gigabit Ethernet was also featured in an external dark fiber application between the ASCI booth and the SGI booth.

The significant contributions that these demonstrations represent were made possible by the many ASCI's technical and commercial partners.

Some of the themes and benefits of this conference were:

- partnering with industry to gain early access to new technology,

- focusing current projects and activities through preparing challenging demonstrations,

- engendering new and evolving partnerships with industry, academia, and the other government labs and agencies,

- discovering and establishing new partnering opportunities,

- highlighting the synergy that results from the tight coupling of networking and communication technologies and organizations,

- providing a stage to professionally interact with colleagues and associates from other organizations in order to challenge and validate our current thinking. 
This paper documents those accomplishments, discusses the details of their implementation, and describes how these demonstrations support DISCOM's strategies in networking

\section{SC ‘98 Networks}

At SC ' 98 , for the third straight year, the three DOE DP Laboratories put together a single integrated research booth. The three Laboratories (Sandia National Laboratories, Los Alamos National Laboratory, and Lawrence Livermore National Laboratory) teamed under the Advanced Strategic Computing Initiative (ASCI) rubric. This year for the first time, the design and operation of the production network was a joint effort of two of the participating organizations. Sandia National Laboratories networking staff and Lawrence Livermore National Laboratory networking staff worked together with the ASCI DISCOM team to provide networking services to the ASCI booth. The production network services included 10/100 Ethernet, OC3 ATM and OC12 ATM. The network interconnection to SCINET was via an OC48 ATM connection. A dark fiber connection was provided to support a proprietary Gigabit Ethernet connection that was utilized by one of the visualization demonstrations. The booth (see Figure 1) was constructed with a raised floor that made the physical layout of the cabling infrastructure much easier than in past years.

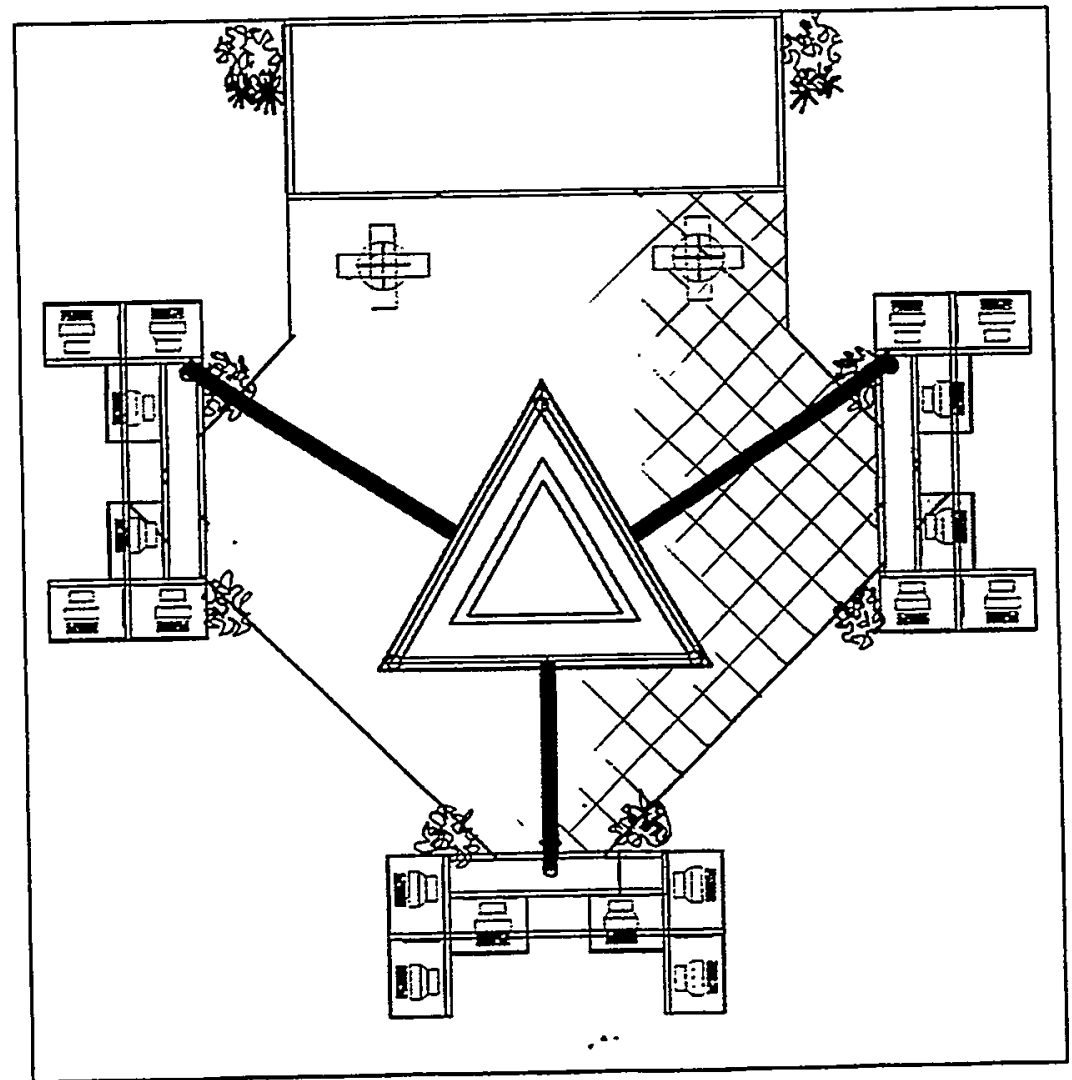




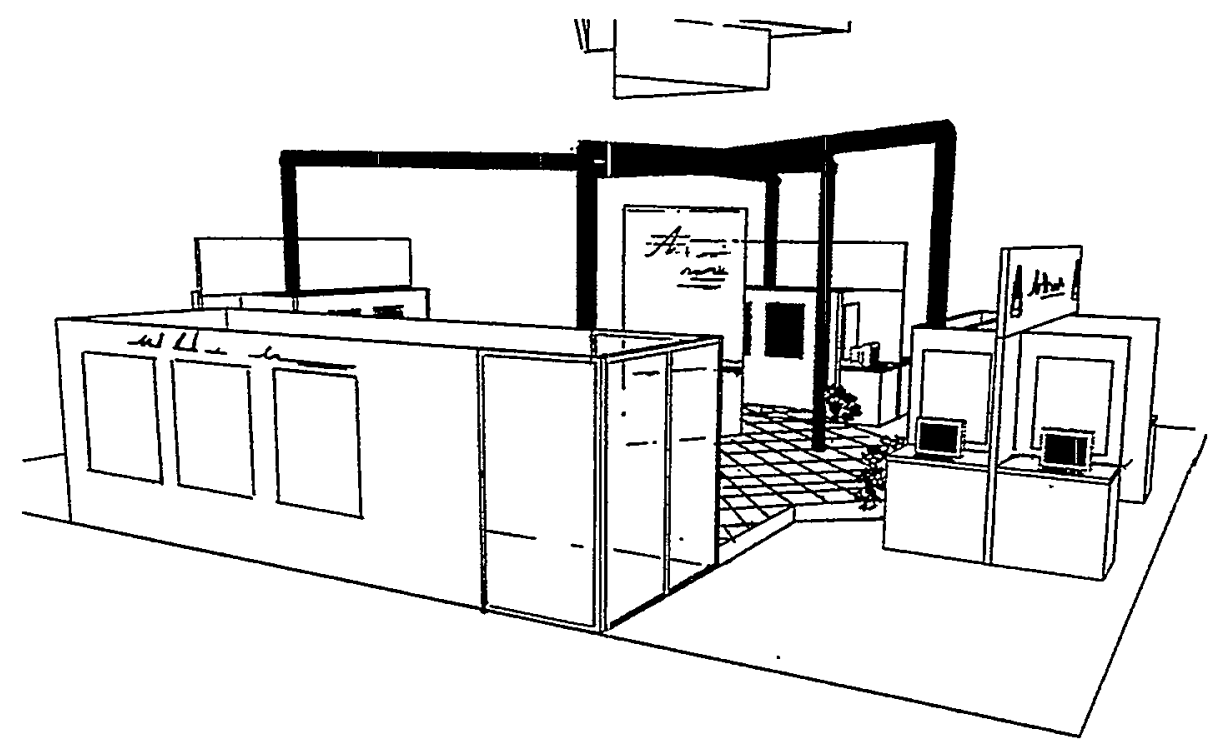

Figure 1: ASCI Booth Layout

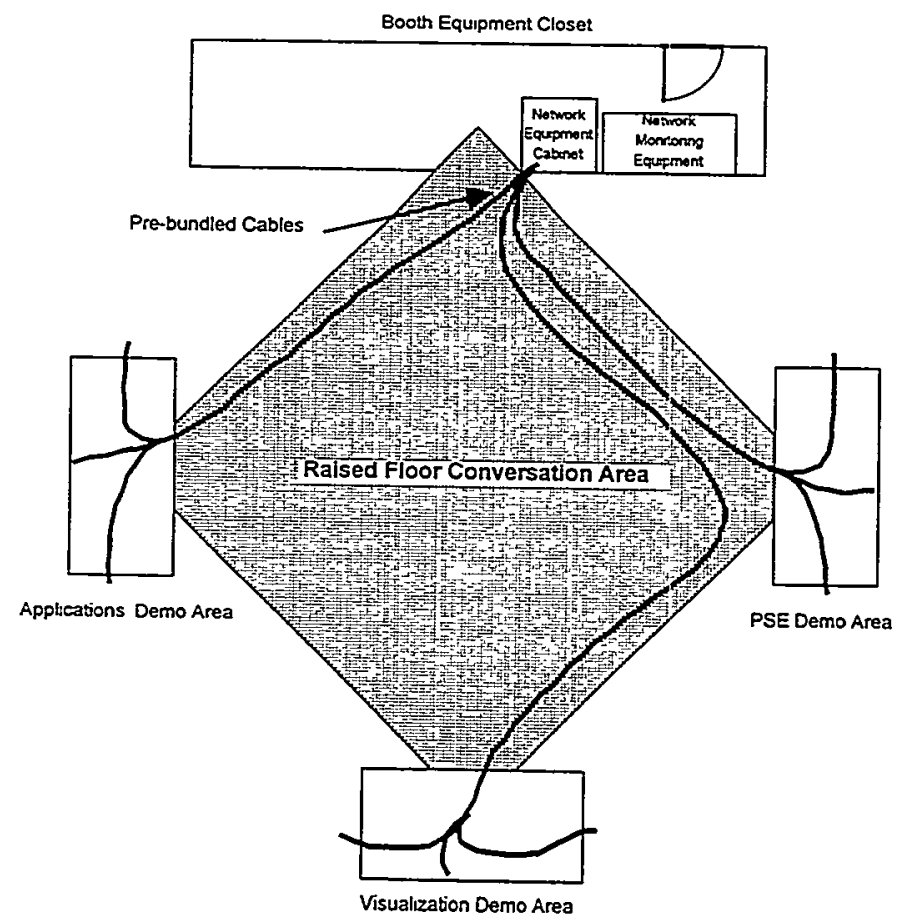

Figure 2: The Cabling Layout

The network infrastructure (see Figure 2) to the demonstration area consisted of category 5 copper cables and multimode fiberoptic cables. The copper cables were grouped together into bundles of eight individual cables. The fiber was put together into a bundle of six fiber pairs. The bundles were then run based on the gathered requirements. As in 
the past years, the requirement prior to getting there were not totally specified. But by going in with two copper bundles and one fiber bundle, almost all the general needs of the demo area were met. In the PSE area, within the booth, a gigabit per second encryption demo and a DFS demo required an additional bundle of fiber cables. A couple of single mode cables were passed into that section because of the possible use of OC48 transport from the encryption demo. The infrastructure planning went very well this year. There was room for improvement, however, in the labeling of cables. This was a past lesson learned that affected us again this year. While all cables were individually marked, the demonstrators did not use this nomenclature and it was difficult to identify the particular fiber or cable that we were interested in once the network came up. This also required that both ends of the cable be looked at prior to making changes. In effect, this doubled the troubleshooting effort.

The booth external connections consisted of an OC48 ATM connection to SCINET that handle the bulk of the demonstration traffic, a 100baseT Ethernet connection to SCINET for protection of the production traffic, and a multimode dark fiber. This dark fiber was for a proprietary gigabit Ethernet connection between an ASCI booth visualization demo and a visualization server in the SGI booth. The proprietary nature of the protocol across the Gigabit Ethernet prevented the use of the booth's general-purpose connection to SCINET.

\section{Network Design}

The production network consisted of Ethernet and ATM links. At the core of the network, were a Cisco 7500 router and a Cisco Catalysts 5500 LAN switch. Three IP networks were attained for the ASCI booth. A Class C IP address space was attained from SCINET to connect most of the booth devices. The second network that we used was a SCINET IP subnet that was a part of the show's ATM network. This network was connected to our booth's router. A few (10) IP addresses where taken from a SCINET Ethernet based network. These addresses were used to connect our network devices management ports and for the DFS demo that had a gigabit ethernet connection. This LAN connection provided the booth with an alternate path in case of a failure on the primary ATM network. It also, gave an out-of-band control channel for the networking devices. A fully redundant network using additional networking gear. Unfortunately, one of the borrowed networking boxes, which had to be built, came to the show in an inoperable state to a non-redundant network design. The configuration of the router can be seen in Figure 3. 


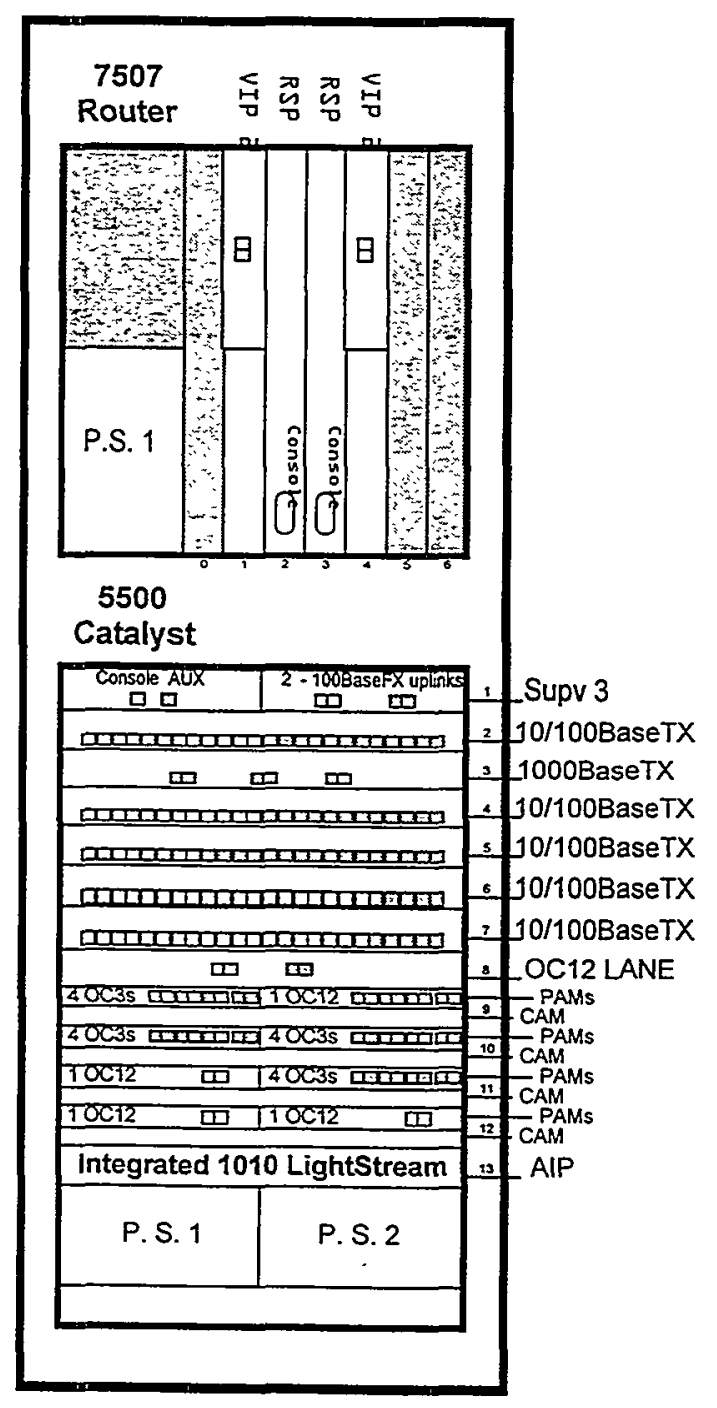

Figure 3: Configuration of Router and Switch in the production ASCI Network

\section{Dense Wave Division Multiplexing Demonstration}

At SC '98 the ASCI DISCOM ${ }^{2}$ project partnered with Lucent Technology to display the capabilities of DWDM, Dense Wave Division Multiplexing, technology. Data generation and aggregation are key elements within the demonstration. FORE Systems and Lucent Technologies both provided high-end ATM switching equipment that aggregated the data before injecting it into the DWDM equipment. Cisco Systems and Sandia National Laboratories provided data networking sources, cluster computers, and test equipment to generate the data directing the demonstration.

DWDM is the technique of passing multiple frequencies of light simultaneously across a single fiber. DWDM is currently being used nationally to dramatically increase the capacity of the installed fiber infrastructure. The requirement to increase capacity is being 
driven by the expanding data transport requirement driven by the explosion in the use of the Internet.

The DISCOM ${ }^{2}$ project views DWDM to be an attractive technology for providing the necessary bandwidth between ASCI computational, visualization and data servers. The parallel nature of DWDM also provides opportunities to extend the massively parallel processing paradigm between geography disbursed computing platforms.

\section{DWDM as Virtual Fiber}

One of the simplest ways to view DWDM technology is as large numbers of virtual fibers that extend from one location to another. The optical signals of the virtual fiber occupy a portion of the actual fiber's optical bandwidth. The current recommendation for this optical channel spacing is 100 gigahertz. This channel specification will easily accommodate OC192, $10 \mathrm{Gbps}$ and should even provide enough space for OC768, 40 Gbps. This channel specification along with characteristic of the currently deployed optical fibers limits the number of channels that can be provided. Current DWDM equipment can provide at least 80 channels of OC48, $2.488 \mathrm{Gbps}$, in this fashion. Advancements in fiber optic cable technologies promises to enlarge the usable space on future fiber greatly.

Even in the simplest applications, DWDM requires several additional components to construct a circuit. The optical inputs for DWDM equipment are specified by three parameters. They are wavelength, bandwidth, and power. The typical input wavelengths used are 1310 or 1550 nanometers. The data bandwidth specification will vary. Currently DWDM equipment is the most efficient when used to pass SONET. This is due in part to the amount of SONET equipment deployed in the market that DWDM is being sold. Broadband modulators, inputs ports that can accept a range of data bandwidth, are available but will usually trail the available SONET rates. Input power specifications should cover a large power range that can be met by using optical amplifiers if the signal need to be boosted or by optical attenuators on circuits where the power level is above the optical receivers power threshold.

\section{DWDM Experiment}

The experiment simulated two large communication centers that need to pass several different types of data. Figure 4 shows the set up of the demonstration. The data types sent across the DWDM system included production internetworking, distributed computing, distance computing, and high-end real-time video transport. Data generation equipment is included to simulate additional loading to the DWDM system. The DWDM system was a Lucent Technologies WaveStar $40 \mathrm{G}$ multiplexer that is capable of multiplexing 16 wavelengths of light onto a fiber. The highest bandwidth that can be carried on a single wavelength is OC48. The maximum amount of data that could be carried in one direction across the system is $39.8 \mathrm{Gbps}$ or a terabyte in 3 minutes and 21 seconds. For the show, each side of the system was provisioned with four OC48, two 
broadband modulators, two OC12, and four OC3. The raw capacity of the total system as configured for the show could provide an aggregate SONET bandwidth equal to OC504 or greater than 26 gigabit per second.

A single piece of equipment does not create a communication network. Aggregation of data to efficiently use or share a large data pipe is required. In this case, a FORE System's ASX4000 40 Gbps ATM switch was used as the data aggregation point in one of the experiment's simulated communication centers. In the other simulated center, a Lucent Technologies' PacketStar Switch provides the data aggregation platform. In addition to these services, two four-node PC clusters were connected directly via DWDM, providing dedicated bandwidth between these machines for high end distributed and distance computing applications. Three OC48 D5 video streams were planned for the system. However, at the last moment, the providers of the video equipment were unable to provide these streams. This service was replaced with FORE Systems MJPEG video units that used OC3 inputs.

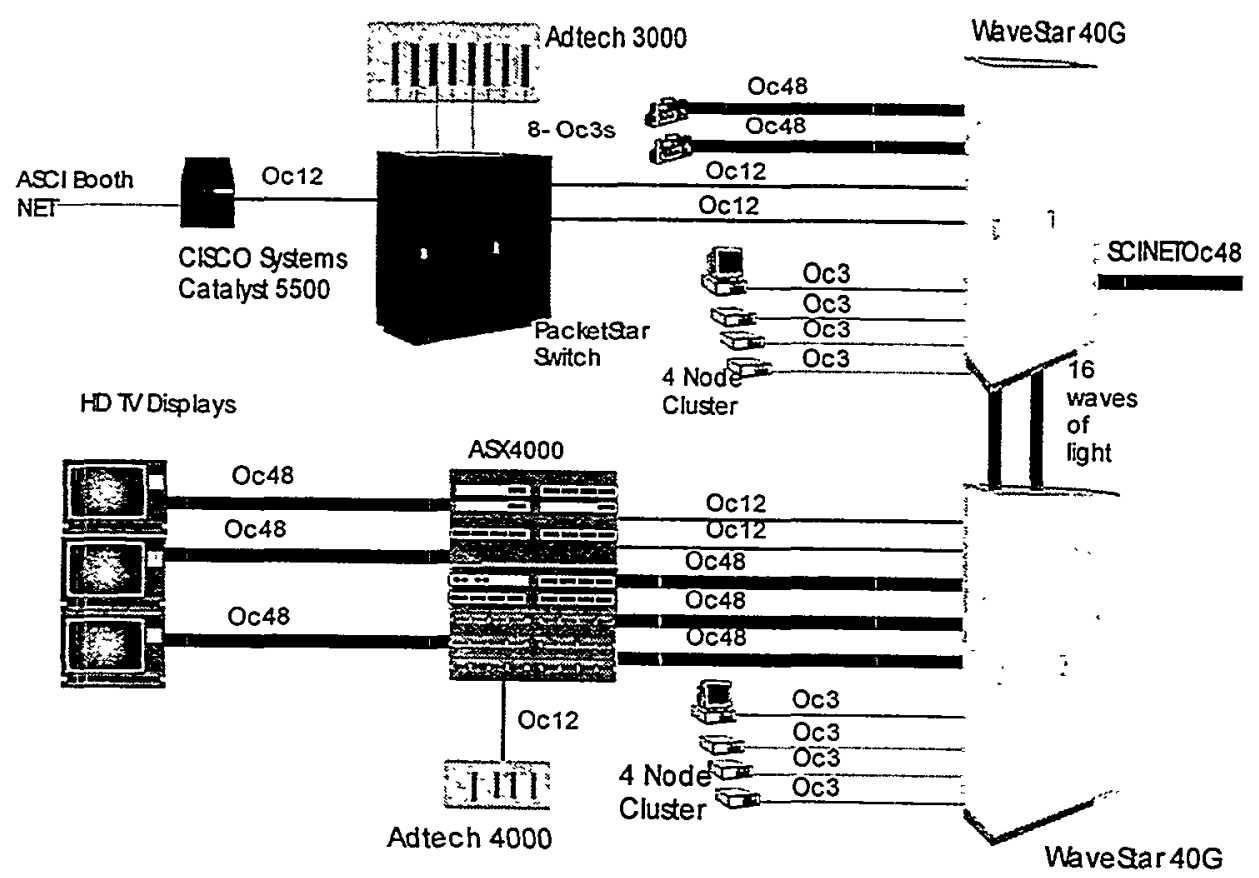

\section{Figure 4: DWDM Demonstration Physical Setup}

The booth's production traffic flowed from the Cisco router into the DWDM Demo. The traffic passed on an OC12 circuit across the system by way of a PVP through the PacketStar switch. The production traffic was passed from an $\mathrm{OC} 12$ circuit to the SCINET provided OC48 and was then passed across the system a second time to arrive at the SCINET OC48 drop. The first test traffic was generated by the ADTECH 3000 . It was capable of generating 1.2 gigabit of ATM cell traffic into the systems. The eight separate OC3 circuits were divided across two of the PacketStar's OC12 circuits and 
the DWDM equipment. The OC12s were delivered to the FORE System ASX4000 and passed into a single OC48 circuit. The OC48 traffic was passed through the DWDM system into an OC48 loopback. The traffic then passed back across the DWDM terminal into a second OC48 port on the ASX4000. The traffic was then passed back out on the original OC48 port where it was added to the original source 1.2 gigabit of data created a full OC48 worth of traffic on that port. The cell stream continued to be passed two more times through the system until the test circuit path $\mathrm{OC} 48$ and $\mathrm{OC} 12$ were full. This traffic routing created a continuous traffic flow of OC204 or 10.5 gigabits of test data through the system. The final destination of the traffic was an Adtech 4000 that could terminate the cell streams. The Adtech 4000 verified that the original Adtech 3000 traffic was being successfully delivered. Additional traffic was added to the system by the ALTA cluster. TCP/IP traffic from each of the cluster nodes was passed across the system. Each of these nodes was able to source and sink greater than $120 \mathrm{mbit} / \mathrm{sec}$ during sustained testing.

During the run of the show, three and a half days, 250 terabytes of data across the DWDM system were exceedingly passed. The test data performance through the system on each day of the show was ramped up. By the end of the show, a terabyte of data through the system passed every 12 minutes. The cell counters in the ASX 4000 when processing this level of traffic rolled over in less than 4 minutes. The traffic as routed didn't affect the other production level traffic running across the network.

\section{Extending the SNL EON with ATM}

During SC '98, the building of a virtual network from the show floor to Sandia National Laboratories, New Mexico was tested. A Permanent Virtual Path (PVP) supplied to us by ESNET and SCINET to our booth was used to build the network. The PVP provided an ATM tunnel to the ASCI Booth network. A port was selected on a LAN switch to be joined into the SNL EON network. The ATM signaling at the edge of the PVP succeeded in creating the LAN connection for the end stations. The ATM path removed all of the IP routers from the data path between SC.'98 and Sandia National Laboratories, NM. The physical path remained practically the same. This eliminated twelve network devices. The resultant roundtrip time from the data was reduced by $25 \%$ or 20 millisecond. The network existed for only 30 minutes prior to the show ending.

\section{Remote Visualization using Compressed ATM Video}

At SC '98, Sandia demonstrated an approach to providing a remote visualization service for its users. This approach used off-the-shelf video compression products to transport visualization images across a wide area network. This demo attempted to implement a high-resolution visualization service by stripping four video streams simultaneously, each representing one quadrant of a $1280 \times 1024$ RGB display. However, due to our inexperience with some of the equipment, the demonstration showed only the central area 
of the RGB monitor. Nevertheless, the demonstration was well-received by a number of labs personnel, as well as other SC ' 98 attendees, and our experience with the equipment allowed us to make an informed choice in the selection of components for a production remote visualization system.

This section of the report describes the SC ' 98 remote visualization demonstration in more detail, including the demonstration setup, demonstration partners, and results.

\section{Setup}

Figure 5 shows the layout for the SC ' 98 demonstration.

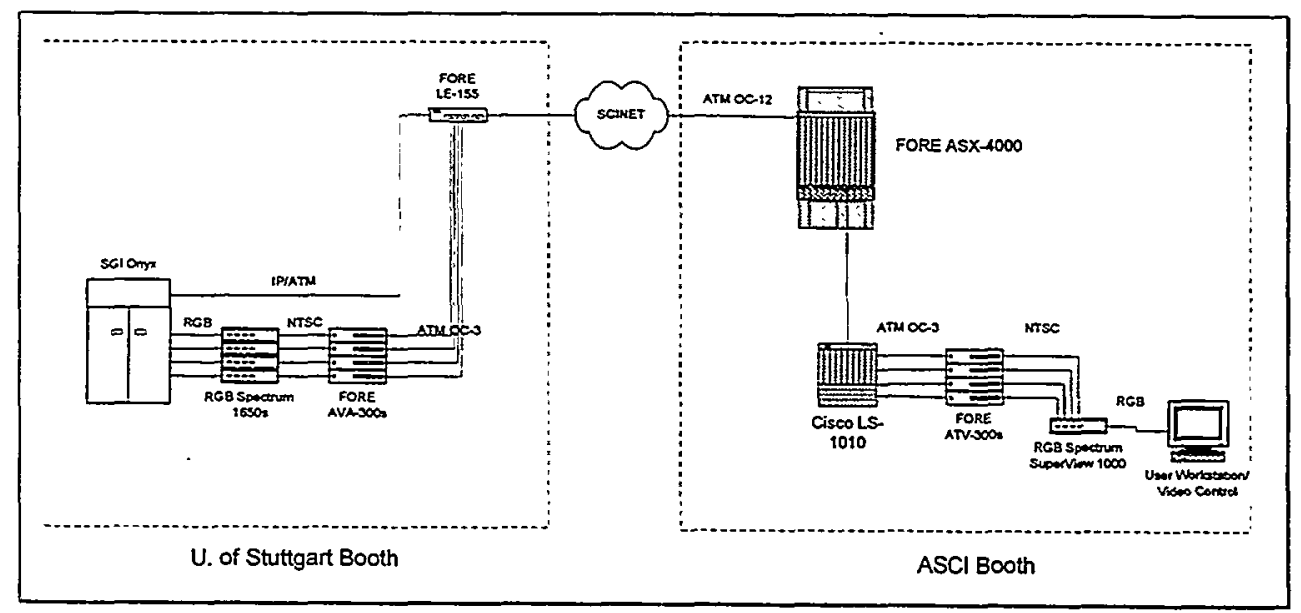

Figure 5: SC '98 Demonstration Setup

In this demonstration, the SGI Onyx in the University of Stuttgart booth was used as the visualization server. The framebuffer data that contains visualization images at $1280 \mathrm{x}$ 1024 resolution is split into four quadrants using the "ircombine" utility, and rendered onto four separate RGB outputs at $640 \times 512$ resolution. Each of these outputs is processed by a separate RGB Spectrum 1650 scan converter to convert the RGB signal into an NTSC signal. The outputs of the scan converters are connected to four FORE Systems AVA-300 ATM video encoders, where the NTSC signals are digitized, . compressed, packed into ATM cells, and transmitted into the SCINET ATM network via a FORE Systems LE-155 low-cost ATM switch.

The remote user in this demonstration "sat" in the ASCI booth. The four ATM video streams entered the ASCI booth through a FORE Systems ASX-4000 ATM switch, and the video streams were switched to a Cisco LS-1010 ATM switch. At this point, the ATM video streams were de-multiplexed to the FORE Systems ATV-300 video decoders, which unpacked the video data contained in the ATM cells, decompressed it, and converted the video data to an NTSC video signal. The four NTSC signals were combined in an RGB Spectrum SuperView 1000 multiple video window system, and were displayed in RGB format on the user's workstation monitor. 
When the user of this system wished to initiate a visualization session, he logged into the visualization server from his workstation. From the visualization server, the user ran a script, which configures the server's framebuffer to display its contents on the four RGB outputs. To display the server's RGB outputs on the user's workstation, the user ran a script on the workstation that tells the video control software to establish the required ATM video circuits. At this point, the user saw the console of the visualization server displayed on his workstation.

The user could start the visualization application. When starting the visualization session, the application was notified that it would receive user control inputs (e.g., keyboard and mouse) and display widgets (buttons, etc.) over a standard X-windows connection. In addition, the application was configured to display rendered visualization output on the framebuffer (which is connected to the user over the ATM video network). This ability to separate user input, widget display, and visualization output required application support.

\section{Results}

Some trouble occurred with some of the RGB Spectrum 1650s, which precluded the conversion of all four RGB outputs to NTSC signals. However, this difficulty was not due to deficiencies in the units-rather, this difficulty was due to our inexperience with these devices. Specifically, the loop-through RGB connectors on the 1650 s needed to be terminated with standard $50 \Omega$ terminators in order to provide a clean synchronization pulse to the scan converter. As a result of our difficulties, it was decided to implement only one video stream, which represented the center quadrant of the Onyx framebuffer. This provided good resolution, but with a limited view of the visualization output.

The reaction received from conference participants was generally very good. Lab users in particular saw the utility of this approach, and had expressed interest in the deployment of a production system based on this concept. However, one impediment to showing this system to users was scheduling time on the Onyx platform for interactive demonstrations. The Onyx platform was shared between users of this demonstration, and users in the University of Stuttgart booth. As a result, there were times when users in the ASCI booth could not directly interact with the visualization code. However, during these times, ASCI users were able to see visualization sessions that were conducted by the Stuttgart users. This allowed the ASCI users to get a sense of the effects of compression on visualization images.

\section{Accelerated Data Transfer with TCP/IP Parallelized FTP}

In the new world of scientific computing, numerous services are delivered to end-users through a plurality of machines with specialized functions. Computation is now often separated from postprocessing in this way, and the mass-storage may no longer be the 
only place for medium-term file caching. The architects of these meta-systems must constantly strive to make greater transport bandwidth available, both present and future. However, rather than simply looking at development of the next hardware, or even firmware, that can speed up throughput among computers, there is an alternate approach-going parallel in both IP connections and TCP-socket applications (e.g., traditional ftp). This achieves substantial, positive scaling in performance with technology you already own and understand how to implement. Although extending the successful model of parallel processing to network data movement may seem obvious, it has been under-exercised until now. Today, in the ASCI project, users are able to put and get files much faster than would be possible with a single, normal circuit of the any physical communication transport technology (which, in our case, today happens to be ATM riding on mostly OC-3 SONET links).

The High-Performance Storage System (HPSS) parallel-ftp, which sits on top of an entire, new data-moving protocol, was the first application to allow separation in network paths, at least on one side. In HPSS, what is known as a storage class, can describe, among other things, an administrator-configured stripe-width across multiple physical volumes. If each of the devices for this media, disk or tape, is mounted on a different mover-service machine, network-parallel data-transfer will automatically occur for the HPSS end. In Figure 6, the HPSS transfer rate graph, shows the initial results of exchanging data between a Sun Microsystems Enterprise 3000, which has a single ATM OC-12 interface to the LAN, and an HPSS configuration that provides direct transfers to IBM 3590 tape in various multiples. An individual IBM RS6000 43P machine with its own network IP address hosts each tape drive, respectively.

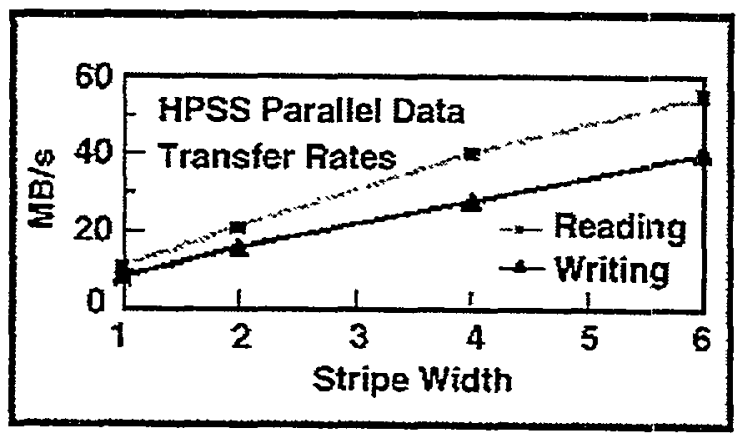

\section{Figure 6: HPSS Transfer Rate Graph}

The special ftp application forks off a stripe-width's compliment of children-each of which has its own TCP socket-and knows how to divide the file data among them to obtain the increased performance. This series ends at a six-wide tape writing stripe, because this is the point where the OC-12 driver for the Solaris could not go any faster. To move to the next level-parallelism on the client side-code was added to the ftp software to offer particular IP-addresses for socket-connections returning from the HPSS mover machines. In the dynamic, this entails: (a) client machines that have additional 
physical ports and logical addresses to offer and (b) movers that can direct their data through these addresses without having the signals go through a single network router. Here the ftp application itself comes both before and after the communications infrastructure, like the chicken and the egg. If the multiple paths cannot be driven in parallel with an equivalent or balanced performance, little or no parallel performance advantage is achieved. One key limitation of this method is, if the data must pass through a normal non-parallel routed infrastructure, then this infrastructure will be an absolute and undermining bottleneck in the system. Figure 7 purposefully shows a switch to highlight this point. However, a parallel networking infrastructure would be worthless without the special data-moving applications.

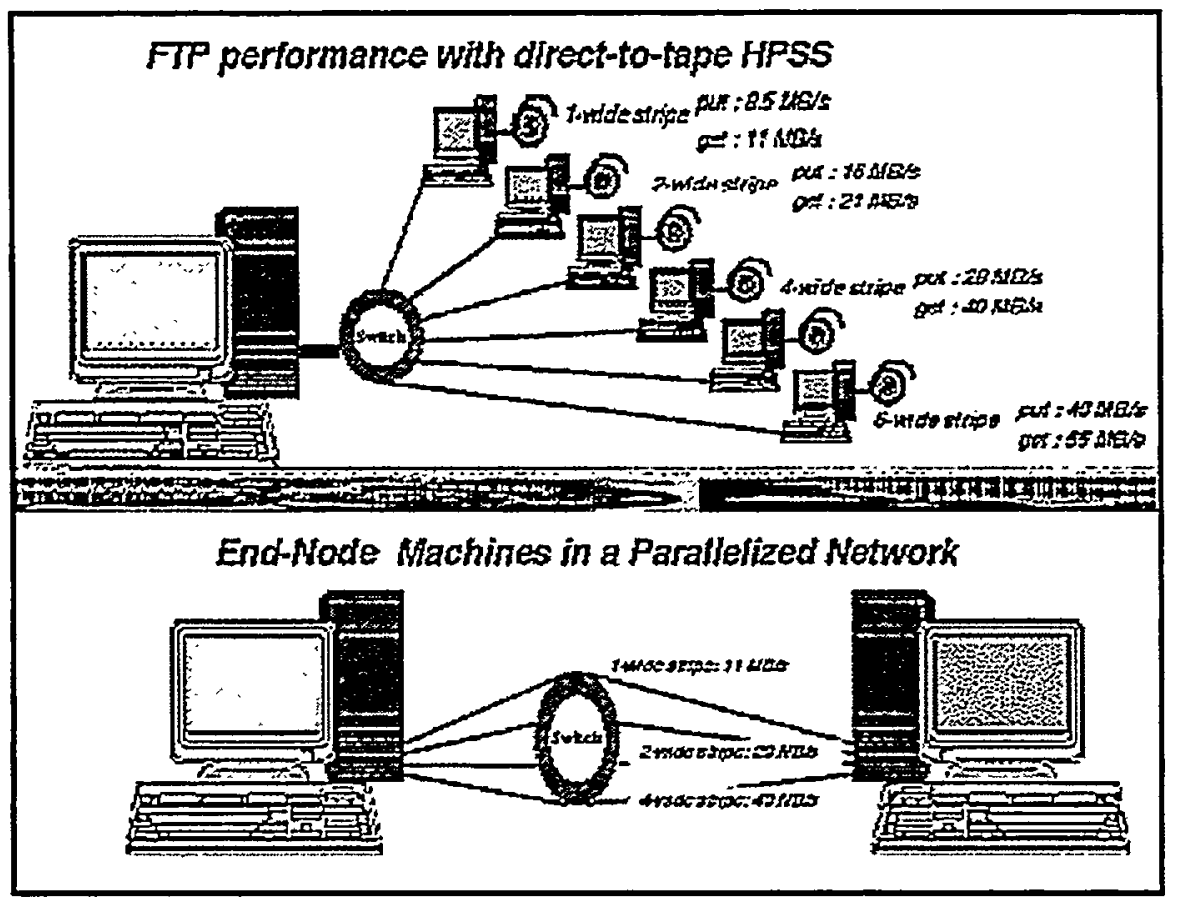

Figure 7: A Parallel Networking Infrastructure

With the capability one is able to increase both ftp throughput and bandwidth, but only when an HPSS system was on one of the ends, a way to deliver data similarly between any two parallel networked machines was needed. For this, as the terminus, a new and exclusive ftp daemon and protocol to derive the results was needed. Something akin to a regular system ftp server had to be augmented to:

- Fork multiple children

- Establish TCP socket connections back to a client for each of them, on disjoint IPaddress paths

- Split or rejoin data correctly, depending on the getting or the putting.

Likewise, these features needed to be added to a generalized client. Once such a tool was implemented, it immediately demonstrated efficacy, and is now being used to pass 
singular large data sets from the parallel disk of the INTEL TERAFLOPS computer directly to SGI Origin machine disk for post-processing. The speeds attained for the increased stripe widths are shown in Figure 8.

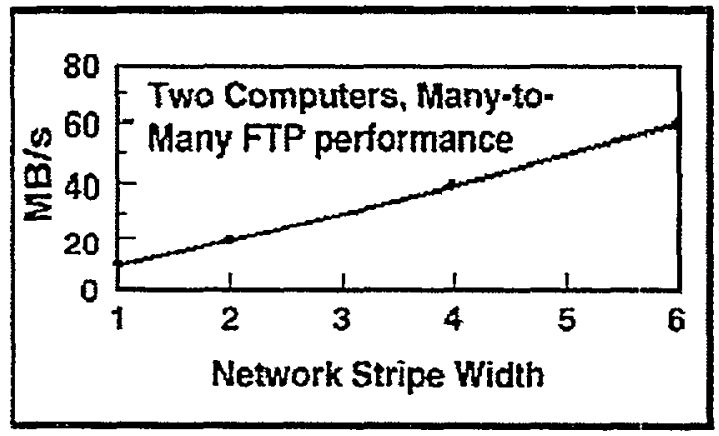

Figure 8: Generalized PFTP Performance

While this is by no means the last word, particularly in answer to the general question of what to do with more and more data, there are tremendous opportunities in leveraging parallelism in network data-movement. Even in cases where singular, very-high speed technology, GSR or POS, is already the normal mode. The multiple-scaling potential could be very beneficial and worth the implementation effort. As is the case with most new computing methodologies, there are trade-offs both for and against implementation. By demonstrating this method at $\mathrm{SC}$ ' 98 , an attempt was made to increase interest within the community associated with high performance data movement. Additionally, this method was presented to scientific computing application developers and users specifically to gauge interest in using PFTP or other parallel data transport and data sharing.

\section{Lessons Learned}

Cable installation, within the booth continues to be a problem area. This year prebundled pre-measured Cat5 UTP cables and duplex OF cables were used in an effort to help minimize this chore. It greatly reduced installation time from previous years but still involved half a dozen people during installation and removal. Pre-bundling the cables required two people, several hours, and a large staging area. Purchasing pre-measured cables required fairly accurate booth dimensions, specific network equipment placement, and time enough to allow for Sandia's procurement cycle. Attaining this kind of information was complicated by the fact that booth dimensions, demo area layouts, and network closet equipment placements were evolving right up to show time. As challenging as this may be, it is the norm for these types of conferences. The network challenge should not be to restrict the participants in these conferences by demanding advance knowledge and commitment in these regards. Rather, the booth network should be as nonrestrictive as possible, offering maximum network flexibility to all booth participants while accommodating the broadest dimensional challenges. 
In the case of SC ' 98 , three application demo areas were established within the booth. A better cabling solution than bundling dedicated Cat5 UTP and duplex OF cables for each of these three areas would have been to install three relatively small port count 10/100 Mbps switches (one in each demo area) and connect them to the network closet with individual duplex OF cables. Three duplex optical fiber cables would have satisfied $90 \%$ of all the requirements in the SC '98 demo areas. In addition, cabling prep time and installation time would have been minimized drastically. Each demo area switch could easily be connected to demo area devices with short Cat5 jumpers. For the few exception demo area devices that require higher bit-rate connections such as Gigabit or ATM, additional individual MM duplex OF cables could be installed along with the demo area duplex OF switch cables. This OF cabling scheme was utilized at SC ' 98 for a few exception devices and it worked nicely.

Generally speaking, installing/removing a duplex OF cable is much easier than a Cat5 UTP cable. This difference is dramatically amplified as the number of cables grows. The fact that OF cable is much more flexible and weighs much less than copper cable should lead us to $\mathrm{OF}$ cable network solutions in the future, wherever possible.

\section{Network Equipment}

The network equipment must be completely reliable. At SC ' 98 , the network equipment was borrowed from Cisco. It was shipped from Cisco directly to the conference. The original network equipment design included two Catalyst 5500s but one chassis arrived DOA. Fortunately, by combining the interface cards in the one functioning Catalyst all booth demonstrators needs were accommodated. Borrowing this equipment was the right thing to do; its combined value was over $\$ 300 \mathrm{~K}$, but shipping it directly to the conference wasn't. In the future, all network equipment should first come to Sandia National Laboratories for testing and configuration. This will increase confidence that the network equipment will function properly at the conference and pre-configuring the equipment at Sandia National Laboratories will speedup network activation.

\section{Internet Access}

External connectivity (from the booth network to the Internet) is always an area of great concern. As sophisticated and advanced as SCINET has become, its network reliability is greatly inferior to the reliability demonstrators have become accustomed to at their respective Lab sites. Ironically, the most pressing demand requiring external connectivity is email access. The demand for email access by attending demonstrators and support staff is equal if not greater than the demand for internal booth connectivity. The goal of the booth network should not be to realign the demonstrators' demands. Rather, the goal of the booth network in this regard should be to establish the best working relationship with SCINET and strive for the best booth Internet access possible. 


\section{Conclusion}

By all measures, the conference proved successful for Sandia National Laboratories. The conference continues to provide a forum to feature a wide variety of state-of-the-art networking and communications technologies and associated applications. This year, the demonstrations benchmarked the feasibility of DWDM and ATM technologies in the networks envisioned by DISCOM. The success stories featured this year were the culmination of work accomplished by many people both within and outside of Sandia National Laboratories. In order to meet the challenging goals of the state-of-the-art networks, many teams were formed that crossed corporate and organizational boundaries. The conference also provided an opportunity to identify future goals and plan joint activities. The teamwork amplified the accomplishments and achievements of all the participants. Similarly, the conference provided the participants an individual opportunity for growth, friendly competition, and professional association. Still, on another level, the conference challenged its participants to take stock of their individual projects and to focus them for the demonstration. In all these ways, Sandia National Laboratories continues to benefit from its participation in Supercomputing.

\section{Acknowledgments}

To put together the activities surrounding the Supercomputing conference takes a large number of talented and dedicated individuals. Without their efforts, Sandia National Laboratories couldn't have accomplished the demonstrations that were done at the conference. We would like to thank the following individuals for their efforts in making ASCI's DISCOM SC '98 networking efforts a success.

SNL

- Thomas V. Archuleta, Arthurine Breckenridge, Joseph Brenkosh, R. Michael Cahoon, Byron Dean, Martha Ernest, Tan Chang "Richard" Hu, John H. Naegle, Mayfann Ngujo, Leonard Stans, Judy Bergman, Ann Hodge, Lyndon Pierson, Perry Robertson, Ed Witzke, Pete Wykopp.

LANL

ESNET

UNM

FORE Systems

Cisco System

Lucent Technologies
- Mitch Sukulli

- Jim Lieghton, Kevin Oberman,

- Karl Gass

- Christina Saraceni, Dave DeBuhr, John Thunhurst, Paul Reisinger,

- Brad Irwin, Mark Bleth

- Don DeMatteo, Ken Evanchik, David Kuenzel, Judy Meester, Rich Peschell, Ray Rancourt, James Verrelle 


\section{References}

[1] Arthurine Breckenridge and Michael O. Vahle. An account of Sandia's research booth at Supercomputing '92: A collaborative effort in high performance computing and networking. Technical Report SAND 93-0224, Sandia National Laboratories, Albuquerque, New Mexico, March 1993.

[2] Steven A. Gossage and Michael O. Vahle. Sandia's research network for Supercomputing '93: A demonstration of advanced technologies for building high performance networks. Technical Report SAND93-3807, Sandia National Laboratories, Albuquerque, New Mexico, December 1993.

[3] Michael O. Vahle, Steven A. Gossage, and Joseph P. Brenkosh. Sandia's network for Supercomputing '94: Linking the Los Alamos, Lawrence Livermore, and Sandia National Laboratories using Switched Multimegabit Data Service. Technical Report SAND 94-3096, Sandia National Laboratories, Albuquerque, New Mexico, December 1994.

[4] Thomas J. Pratt, Michael O. Vahle, and Steven A. Gossage. Sandia's network for Supercomputing '95: Validating the Progress of Asynchronous Transfer Mode (ATM) Switching. Technical Report SAND 96-0820, Sandia National Laboratories, Albuquerque, New Mexico, April 1996.

[5] Thomas J. Pratt, Luis G. Martinez, Michael O. Vahle, Thomas V. Archuleta. Sandia's network for Supercomputing '96 Linking Supercomputers in a Wide Area Asynchronous Transfer Mode (ATM) Network. Technical Report SAND 97-0748 UC-705 Sandia National Laboratories, Albuquerque, New Mexico, April 1997.

[6] Thomas J. Pratt, Luis G. Martinez, Michael O. Vahle, Thomas V. Archuleta, Vickie K. Williams. Sandia Network for SC97: Supporting Visualization, Distributed Cluster Computing, and Production Data Networking with a Wide Area High Performance Parallel Asynchronous Transfer Mode (ATM) Network. Technical Report SAND 981154, Sandia National Laboratories, Albuquerque, New Mexico, May 1998. 
Distribution

Lawrence Livermore National Laboratory

Wayne Butman, L-072

P. O. Box 808

Livermore, CA 94550

Lawrence Livermore National Laboratory

David P. Wiltzius, L-060

P. O. Box 808

Livermore, CA 94550

Los Alamos National Laboratory

Attn: Stephen C. Tenbrink

CIC-5 Network Engineering

Los Alamos, NM 87545

$1-0318$ A. Breckenridge, 9215

1 - 0318 G. S. Davidson, 9201

1 - 0321 W. J. Camp, 9204

1 - 0321 A. L. Hale, 9224

1 - 0467 R. J. Detry, 2900

1 - 0660 W. D. Swartz, 4619

1 - 0661 M. H. Pendley, 4612

1 - 0741 S. G. Varnado, 6200

1 - 0801 M. J. Murphy, 4400

1 - 0803 J. F. Jones, Jr., 4600

10 - 0806 M. O. Vahle, 4616

1 - 0806 J. P. Brenkosh, 4616

1 - 0806 J. M. Eldridge, 4616

1 - 0806 M. J. Ernest, 4616

1 - 0806 S. A. Gossage, 4616

$1-0806$ T. C. Hu, 4616

1 - 0806 J. A. Hudson, 4616

25 - 0806 L. G. Martinez, 4616

1 - 0806 J. H. Naegle, 4616
1 - 0806 L. G. Pierson, 4616

$25-0806$ T. J. Pratt, 4616

1 - 0806 J. A. Schutt, 4616

1 - 0806 L. Stans, 4616

1 - 0806 T. D. Tarman, 4616

1 -0806 E. L. Witzke, 4616

1 - 0806 C. D. Brown, 13221

1 - 0807 I. C. Alexander, 4417

1 - 0807 S. D. Nelson, 4417

1 - 0807 R. M. Cahoon, 4418

1 - 0812 M. R. Sjulin, 4914

10 - 0812 R. L. Adams, 4914

1 - 0812 F. J. Castelluccio, 4914

1 - 0812 L. F. Tolendino, 4914

1 - 0812 B. C. Whittet, 4914

1 - 0812 G. A. Yonek, 4914

1 - 0820 P. Yarrington, 9232

1 - 0826 J. D. Zepper, 9136

1 - 0841 P. J. Hommert, 9100

1 - 1109 K. S. McCurley, 9224

1 - 9003 D. L. Crawford, 9900

1 - 9006 J. Costa, 9903

1 - 9011 J. C. Berry, 8910

1 - 9011 J. M. Brandt, 8910

1 - 9011 H. Y. Chen, 8910

1 - 9011 P. W. Dean, 8903

1 - 9011 R. D. Gay, 8920

1 - 9012 C. L. Yang, 8920

1 - 9012 F. T. Bielecki, 8930

1 - 9012 J. A. Friesen, 8990

1 - 9037 J. A. Hutchins, 8930-1

2 - 0899 Technical Library, 4916

2 - 9019 Central Technical Files, 8940

2 - 0619 Review \& Approval Desk, 00111 for DOE/OSTI 